Copyright (C) 2016 IEEE. Personal use of this material is permitted. Permission from IEEE must be obtained for all other uses, in any current or future media, including reprinting/republishing this material for advertising or promotional purposes, creating new collective works, for resale or redistribution to servers or lists, or reuse of any copyrighted component of this work in other works. 


\title{
A Combined High Efficiency Region Controller to Improve Fuel Consumption of Power-Split HEVs
}

\author{
Shane Overington, Member, IEEE and Sumedha Rajakaruna, Senior Member, IEEE
}

\begin{abstract}
An improved controller for the energy management system of a power-split hybrid electric vehicle is developed with the objectives of minimizing fuel consumption and improving drivability. Considering the specific application of vehicles plying on scheduled trips such as public transport, the paper assumes that the controller is privileged with a priori knowledge of the estimated total tractive energy requirement and the duration of the journey. In comparison to a recently introduced constant high efficiency region based controller, the paper demonstrates that further reductions in fuel consumption can be achieved under certain driving cycles by limiting the internal combustion engine operation to a dynamically varying high efficiency region and adopting state of charge swing control for the battery energy storage. The frequency of engine on/off is therefore directly decided by the size of the energy storage, allowable swing of the state of charge and the tractive energy required. Performances of the constant high efficiency region and dynamic high efficiency region controllers are compared through simulations against the existing controller of a commercial vehicle. The results reveal the dynamic high efficiency region controller outperforms in fuel consumption over the other two controllers in highway style drive scenarios. Therefore, to minimize fuel consumption whilst improving drivability under all drive scenarios this paper proposes to combine the constant high efficiency region controller with the dynamic high efficiency region controller such that the best features of both controllers can be utilized.
\end{abstract}

Index Terms-Hybrid, electric vehicles, power-split, internal combustion engine, fuel consumption, state of charge swing, high efficiency, energy management, highway driving.

\section{NOMENCLATURE}

$\begin{array}{ll}D_{e} & \text { ICE on/off. } \\ E_{e s s} & \text { ESS energy }(\mathrm{J}) . \\ E_{f} & \text { ICE fuel energy (J). } \\ E_{L} & \text { Tractive energy (load, J). } \\ E_{t o t a l} & \text { Planned route total tractive energy (J). } \\ f_{e} & \text { ICE on/off frequency. } \\ J & \text { Objective function. } \\ P_{a v} & \text { Average tractive power (load, W). } \\ P_{e} & \text { Internal combustion engine output power }(\mathrm{W}) . \\ P_{e, a v} & \text { Average ICE output power }(\mathrm{W}) . \\ P_{e, a v} & P_{e, a v} \text { optimal operating line vector. } \\ P_{e s s} & \text { Energy storage system output power }(\mathrm{W}) . \\ P_{I} & \text { Power required to overcome rotational moment of } \\ P_{L} & \text { inertia (W). } \\ P_{m / g} & \text { Tractive power (load, W). } \\ P_{\eta e, \text { low }} & \text { Motor/generator power (load side, W). } \\ & \text { ICE output power corresponding to } \eta_{e, \text { low }}(\text { varies } \\ & \text { with } T_{e} \text { and } \omega_{e}, \text { W) }\end{array}$

Copyright ( 2015 IEEE. Personal use of this material is permitted. However, permission to use this material for any other purposes must be obtained from the IEEE by sending a request to pubs-permissions@ieee.org.

The authors are with Department of Electrical and Computer Engineering, Curtin University, GPO Box U1987, Perth, Western Australia 6845, Australia. (email: shane.overington@gmail.com, and S.Rajakaruna@ @urtin.edu.au

$\begin{array}{ll}R_{\text {int }} & \text { ESS internal resistance }(\Omega) . \\ S O C & \text { ESS state of charge. } \\ S O C_{\text {final }} & \text { SOC at the end of driving. } \\ S O C_{\text {high }} & \text { SOC high threshold. } \\ S O C_{\text {init }} & \text { SOC at the start of driving. } \\ S O C_{\text {low }} & \text { SOC low threshold. } \\ T_{e} & \text { ICE output torque (Nm). } \\ t_{\text {off }} & \text { ICE off time (s). } \\ t_{\text {on }} & \text { ICE on time (s). } \\ t_{t r i p} & \text { Planned route estimated time of arrival (s). } \\ V & \text { Vehicle velocity (m/s). } \\ V_{e s s} & \text { ESS terminal voltage (V). } \\ V_{o c} & \text { ESS open circuit voltage (V). } \\ \Delta E & \text { Energy balance between ICE and ESS with } \\ & \text { respect to } E_{L}(\mathrm{~J}) . \\ \eta_{c} & \text { Coulomb efficiency. } \\ \eta_{d} & \text { Drivetrain efficiency. } \\ \eta_{e} & \text { ICE efficiency. } \\ \eta_{e, l o w} & \text { Lowest allowable efficiency contour for the high } \\ \bar{\eta}_{e, l o w} & \text { efficiency region. } \\ \eta_{e s s} & \eta_{e, l o w} \text { optimal operating line vector. } \\ \eta_{m / g} & \text { ESS efficiency. } \\ \eta_{s y s} & \text { Motor/generator efficiency. } \\ \eta_{t} & \text { Overall system efficiency. } \\ \omega_{e} & \text { Transmission efficiency. } \\ & \text { ICE output speed (rad/s). }\end{array}$

\section{INTRODUCTION}

World energy consumption is projected to increase by $56 \%$ between 2010 and 2040 [1]. Among the many different sectors, transportation accounts for the second largest percentage at $26 \%$ in 2010 [1]. Alternative means for transportation are therefore highly sort after especially in light of the high fuel consumption of today's conventional vehicles [2]. The power-split hybrid electric vehicle (HEV) has been identified as having one of the greatest potentials for minimizing fuel consumption and thereby greenhouse gas emissions whilst meeting the demands of driving [3-7]. The controller of the energy management system (EMS) is the brain of such hybrid vehicles that determines the critical performance specifications such as fuel consumption and drivability. One of the key decisions it has to make is in deciding the relative contributions of energy through the energy storage system (ESS) and the internal combustion engine (ICE) under different driving conditions.

Performance of HEV is measured by reduced fuel consumption, reduced emissions and drivability. Firstly, a reduction in the fuel consumption over standardized drive cycles constitutes a savings in fuel and a reduction in $\mathrm{CO}_{2}$ emissions [3, 4, 8-10]. Secondly, drivability in $\mathrm{HEV}$ is measured with respect to the total number of ICE events [8]. The starting and stopping of the ICE identifies one ICE event during driving, whereby a minimum number of ICE events are desirable [8]. This also aligns with the desire for more 
efficiently controlled and longer lasting ESS [11]. Recent literature has discussed the adverse effects on the expected operating life of ESS such as battery energy storage in HEV due to the increased number of charge/discharge cycles [11]. One of the methods for improving battery life expectancy while reducing fuel consumption in HEVs is through the control of state of charge (SOC) swing of the battery. In SOC swing control, SOC of the battery is restricted to vary between a specified maximum value $\left(S O C_{h i g h}\right)$ and a specified minimum value $\left(S O C_{\text {low }}\right)$ [12]. In addition to resulting in controlled charge/discharge events of the battery leading to possible increased life expectancy, SOC swing control also paves a way to resolve the issues caused by frequent ICE on/off events, which contribute to poor drivability [8]. On the other hand, restricting the ICE events in this manner can lead to performance issues, especially during ICE shutdown where high tractive power requests are solely supplied by the battery energy storage $[3,13]$.

SOC swing control is typically utilized in series connected HEV where there is a distinct ICE on/off period for charging and discharging of the ESS $[4,9]$. However, series connected topologies have demonstrated increased power losses in the powertrain due to the high number of energy conversion stages, limiting the potential for fuel savings [14]. Implementation of SOC swing control strategies in parallel connected vehicles are restricted by the direct coupling of the powertrain to the drivetrain, which ultimately lowers average ICE efficiency and increases fuel consumption [14]. The power-split configuration on the other hand utilizes the planetary gear set (PGS) to increase the flexibility in operation of the powertrain, by offering both mechanical (parallel) and electrical (series) energy paths. It therefore has the best potential for fuel consumption improvement and is utilized in this paper as the topology for implementing the proposed controller [6].

The authors have recently introduced a rule-based optimizing controller referred to in this paper as the Constant High Efficiency Region (CHER) controller [10], where the ICE is always operated within a pre-defined constant high efficiency region on the torquespeed plane. The EMS controller directly regulates the operation of ICE on the torque-speed plane while the SOC of the ESS is regulated by a $S O C_{\text {target }}$ charge strategy. It has been shown to perform better than a stock controller resulting in reduced fuel consumption under different drive cycles, however the CHER controller still operates with a high number of ICE on/off events. In this paper it is aimed to demonstrate by utilizing a novel SOC swing controller for the ESS coupled with the novel Dynamic High Efficiency Region (DHER) control for the ICE, further reductions in fuel consumption and ICE on/off events are possible under certain driving conditions. DHER control dynamically varies the high efficiency region within which the ICE is constrained to operate by utilizing the most recently updated information about the trip. However, the simulation results reveal that the benefits of this controller diminish in drive cycles consisting with frequent operations at low vehicle speeds. In retaining the best of both CHER and DHER controllers, the paper therefore presents a 'Combined HER' controller that can yield low fuel consumption and reduced ICE events under all driving conditions.

In order to aid the energy management system of HEV, global positioning systems (GPS) and intelligent transportation systems (ITS) offer the potential for planning drive routes ahead of time [15, 16]. The a priori information referred to in this paper for the control of HEVs stems from the existence of predictive control [7] and information available to the energy management system controllers

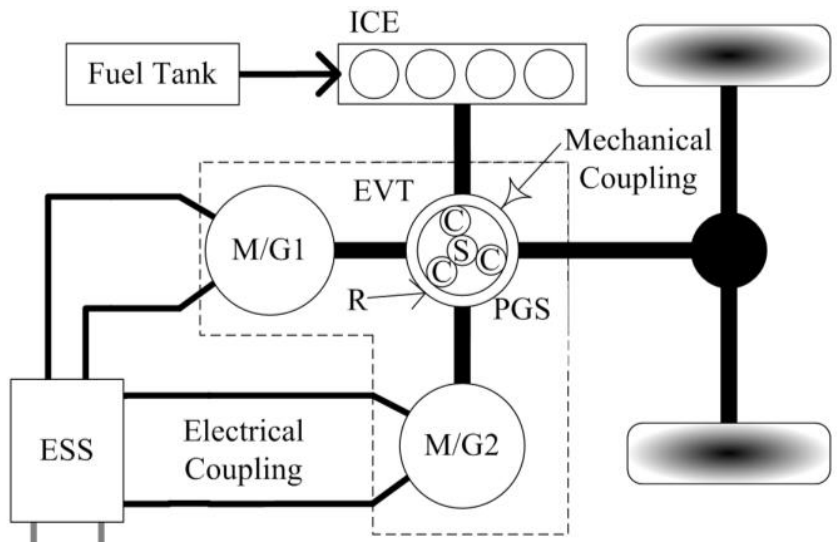

Fig. 1. Toyota Prius 2010 vehicle architecture.

from GPS and ITS [15, 17]. Predictive controllers aim to use past and present trip information in real-time optimization routines to determine the future operations of the $\operatorname{HEV}[11,16]$. The main drawback of predictive controllers during optimization is the large amounts of information that need to be analyzed in real-time [6,9], while higher accuracy is the main advantage. The high processing power needed in predictive controlling can be significantly reduced if some of the key information of the planned future trip can be passed to the EMS controller as 'a priori knowledge' [16, 17]. The SOC swing controller with DHER control of the ICE, described in this paper utilizes a priori information of a planned route such as the tractive energy requirement $\left(E_{\text {total }}{ }^{\prime}\right)$ and trip time $\left(t_{\text {trip }}{ }^{\prime}\right)$, in order to minimize the fuel consumption in HEVs. This a priori information is expected to be available before beginning and also during the trip. One or many vehicles offering a transportation service such as a delivery service or public transport for example may operate over planned routes, with a consistent frequency or number of stops while operating to a strict schedule for its customers. In such preplanned trips, the proposed controller using the readily available a priori information has the potential to yield better performance than previously published controllers.

This paper is organized as follows; Section II outlines the test bench utilized for the comparison of the EMS controllers. Section III defines the CHER and DHER controllers separately before introducing the Combined HER controller. Section III then leads into an outline of the procedure for calculating and implementing the Combined HER controller. Section IV compares the efficacy of the three controllers with respect to the existing controller of the Toyota Prius 2010 over three standardized drive cycles using simulation results from the ADVISOR software. Finally the contributions of this paper are summarized in Section V.

\section{TEST BENCH}

The proposed controller is implemented on the Toyota Prius 2010 as shown in Fig. 1 with specifications outlined in Table I. The Toyota Prius 2010 is a power-split HEV with a controller for the existing vehicle (Orig. in Table II) developed in comparison to measured data and defined and verified in [10]. Fig. 1 identifies the main components of the Toyota Prius power-split topology, consisting of an ESS, an ICE and two motor/generators (M/G1 and $\mathrm{M} / \mathrm{G} 2$ ). The ICE, M/G1 and M/G2 are all coupled to a PGS which connects to the wheels of the vehicle via a final drive. The PGS has the ICE connected to the carrier (C) gears, M/G1 connected to the sun gear $(\mathrm{S})$, and $\mathrm{M} / \mathrm{G} 2$ connected to one side of the ring $(\mathrm{R})$ gear 
with the final drive coupled to the opposite side of the ring gear. Table I lists the vehicle specifications for the Toyota Prius 2010 vehicle. The study presented in [10] verifies the accuracy of the Toyota Prius 2010 test bench.

\section{EMS CONTROLLER}

\section{A. Constant High Efficiency Region Controller}

According to the CHER controller [10], operation of the ICE is restricted to a certain region in the torque-speed plane defined by the lowest allowable efficiency $\left(\eta_{e, \text { low }}\right)$ contour. Fig. 2 , for example, shows a high efficiency region on the torque-speed plane defined by $\eta_{e, \text { low }}=32 \%$. As discussed in [10], both power balancing and variable speed control are utilized to achieve improved efficiency for torque and speed requests that lie outside the high efficiency region. Both of these approaches to improving the operating efficiency of the ICE are illustrated in Fig. 2. The optimal operating line shown in Fig. 2 identifies the maximum efficiency for which the ICE is capable of producing a given power level $\left(P_{e}\right)$, which is enforced through variable speed control. This means that all power requests from the ICE (in a power-split topology) will be met with the ICE operating somewhere on the optimal operating line of Fig. 2. For example, variable speed control is the movement from a power request of $P_{e l}=T_{e l}\left(\dot{m}_{f l}\right) \omega_{e l}$ to $P_{e l}=T_{e 3}\left(\dot{m}_{f 3}\right) \omega_{e 3}$, with no change in operating power requested from the ICE. Power balancing on the other hand, requires varying the ICE operating power and relying on the ESS to absorb the difference (from $P_{e l}=T_{e l}\left(\dot{m}_{f l}\right) \omega_{e l}$ to $\left.P_{e 2}=T_{e 2}\left(\dot{m}_{f 2}\right) \omega_{e 2}\right)$. Additionally, [10] introduces the two variables $P_{\eta e, \text { low }}{ }^{-}$and $P_{\eta e, \text { low }}{ }^{+}$which as identified in Fig. 2 define the boundary for operation within the high efficiency region (relative to $\eta_{e, \text { low }}$ ) along the optimal operating line.

\section{B. Performance Limitations of CHER Controller}

The CHER controller presented in [10] is utilized for comparison of the proposed controller's ability to minimize fuel consumption. As mentioned the CHER has a predefined value for $\eta_{e, l o w}$ where the EMS controller balances the energy requirement of the load between the ESS and ICE. It is important to note that the CHER controller has frequent ICE on/off as a result of using predefined rules for controlling the SOC of the ESS [10]. The CHER controller while capable of maintaining ESS SOC and reducing fuel consumption requires calibration, pre-determining the optimal high efficiency region for the vehicle it is utilized on.

It was noticed during further analysis of the CHER controller that for highway driving (e.g. HWFET) fuel savings were minimal compared to those found for city driving (e.g. UDDS and NEDC) [10]. An initial investigation realized the modifications proposed here for the CHER controller. These modifications lead to greater fuel savings in the CHER controller on the considered highway driving scenario. During highway driving the tractive power required to maintain constant speed is not as large as the power required for acceleration. Given that any additional power generated by the ICE at high vehicle velocity must be absorbed by the ESS, if the power generated by the ICE is larger than the requested demand the energy is unnecessarily wasted. Therefore the aim during highway driving for the CHER controller is to mitigate losses by offering a more manageable average power level.

\section{Dynamic High Efficiency Region Controller}

TABLE I

VEHICLE SPECIFICATIONS

Component Parameter

ICE peak power $(\mathrm{kW})$

ICE peak torque $(\mathrm{Nm})$

ICE peak efficiency (\%)

$\mathrm{M} / \mathrm{G} 1$ peak power $(\mathrm{kW})$

$\mathrm{M} / \mathrm{G} 2$ peak torque $(\mathrm{Nm})$

$\mathrm{M} / \mathrm{G} 2$ peak power $(\mathrm{kW})$

$\mathrm{M} / \mathrm{G} 2$ peak torque $(\mathrm{Nm})$

Transmission

ESS
Toyota Prius 2010

$73($ @ $5200 \mathrm{rpm})$
$142(@ 4000 \mathrm{rpm})$
$36^{*}$
42
$84.7^{*}$
60
207
EVT
MH 201.6 V and $7.2 \mathrm{Ah}$

'*' indicates estimated values for simulation purposes

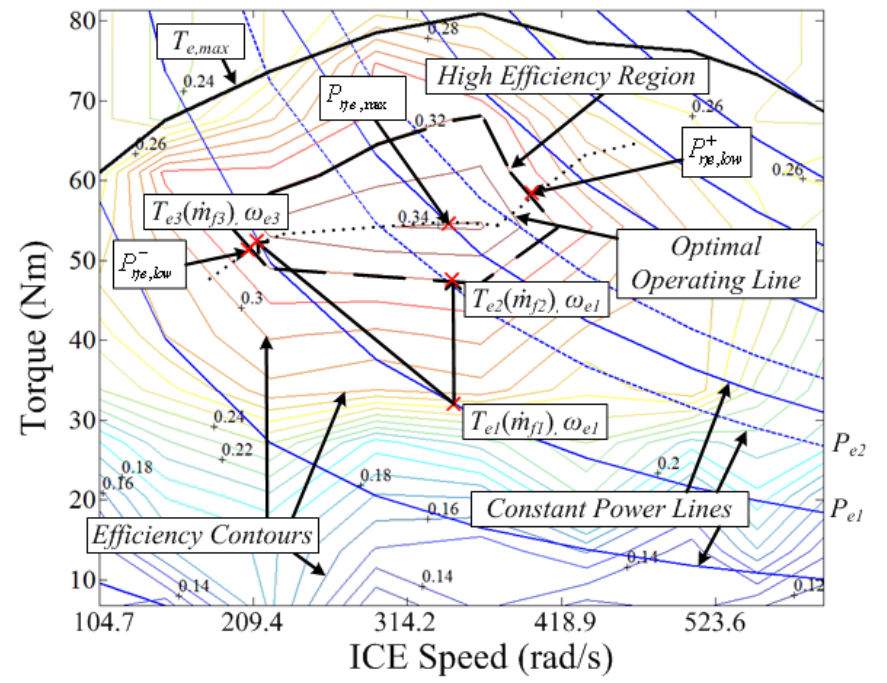

Fig. 2. ICE performance map similar to that presented in [10], shifting of $T_{e l}\left(\dot{m}_{f l}\right), \omega_{e l}$ to $T_{e 3}\left(\dot{m}_{f 3}\right), \omega_{e 3}$ demonstrates variable speed control (no power increase), and shifting from $T_{e l}\left(\dot{m}_{f 1}\right), \omega_{e l}$ to $T_{e 2}\left(\dot{m}_{f 2}\right), \omega_{e l}$ demonstrates power balancing (increase in power $P_{e 1}$ to $P_{e 2}$ ).

As a means of overcoming the stated deficiencies of the CHER controller this paper investigates DHER control of the ICE in combination with a SOC swing charge strategy for the ESS. The DHER introduces a varying value of $\eta_{e, \text { low }}$ throughout driving based on the most updated information of driving conditions. Referring to Fig. 2, the DHER thereby allows for the change of the $P_{\eta e, l o w}{ }^{-}$and $P_{\eta \text { elow }}{ }^{+}$values relative to $\eta_{e, l o w}$. The SOC swing charge strategy ensures the SOC of the battery is controlled to within a specified range, defining the minimum acceptable level $\left(S O C_{l o w}\right)$ and maximum acceptable level $\left(S O C_{\text {high }}\right)$ of SOC for the battery. This helps to improve the life of the battery, improves drivability and assures energy availability from the battery under all driving conditions. Instead of having a single $\eta_{e, \text { low }}$ value for ICE operation (as defined in [10]) the CHER controller as presented in this paper is modified to include two alternative $\eta_{e, \text { low }}$ values with respect to vehicle velocity:

$$
\eta_{e, \text { low }}=\left\{\begin{array}{l}
\eta_{e, \text { low } 1}, \text { for } V>V_{1} \\
\eta_{e, \text { low } 2}, \text { for } V \leq V_{1}
\end{array}\right.
$$

where $\eta_{e, \text { low } 2}>\eta_{e, \text { low } 1}$ since the ICE should allow more flexible operation at higher vehicle velocity. The objective function defined for operation of the ICE in the DHER controller is: 


$$
\text { Objective: minimize } J=E_{f}\left(t_{o n}\right)
$$

Where $E_{f}\left(t_{o n}\right)$ is the fuel energy consumed during the on period $\left(t_{o n}\right)$ of the ICE, with respect to the following constraints:

$$
\text { Subject to: }\left\{\begin{array}{l}
P_{e s s, \text { min }}(t) \leq P_{e s s}(t) \leq P_{e s s, \text { max }}(t) \\
P_{m / g, \text { min }}(t) \leq P_{m / g}(t) \leq P_{m / g, \text { max }}(t) \\
P_{\eta e, l o w}^{-} \leq P_{e}(t) \leq P_{\eta, \text { low }}^{+} \\
P_{e s s, \text { max }}(t) \geq \eta_{m / g} \eta_{c} P_{\eta e, l o w}^{-} \\
E_{L}+\frac{1}{2} \Delta E>\eta_{d} \eta_{t} \eta_{e} E_{f}\left(t_{o n}\right) \\
S O C_{l o w} \leq S O C(t) \leq S O C_{\text {high }}
\end{array}\right.
$$

In particular (3) defines the maximum and minimum power ratings of ESS $\left(P_{e s s}\right)$ and $\mathrm{M} / \mathrm{G}\left(P_{\mathrm{m} / \mathrm{g}}\right)$, which determine the operating capability during ICE shutdown, then $\eta_{e}, \eta_{t}, \eta_{d}, \eta_{m / g}, \eta_{c}$, are efficiencies of the ICE, transmission, final drive, M/G, and ESS (coulomb) respectively and the inequality of $E_{L}+1 / 2 \Delta E>\eta_{d} \eta_{t} \eta_{e} E_{f}(t)$ is the threshold for ICE shutdown as discussed in Section III D. The final constraint of (3), is to limit the SOC swing of the battery between $S O C_{\text {low }}$ and $S O C_{\text {high }}$.

Equations (2) and (3) define the problem for the investigated power-split configuration. However, in the case of the DHER controller presented in this paper the solution needs to optimize the selection of the high efficiency region of the ICE $\left(\eta_{e, \text { low }}\right.$ and therefore $P_{\eta \text {,low }}{ }^{-}$and $P_{\eta e, l o w}{ }^{+}$) in order to meet the drivetrain load requirements during ICE operation. Firstly, the EMS block diagram of Fig. 3 is inserted as the EMS controller block defined in [10], secondly, command signals are followed by an apostrophe ('), while measured signals are not. Fig. 3 indicates that the DHER controller utilizes requested load and past recorded information of the vehicle in order to determine ICE on/off $\left(D_{e}\right)$ and output torque $\left(T_{e}\right)$ and speed $\left(\omega_{e}\right)$. In addition, the EMS controller utilizes an expected average value of drivetrain load $\left(P_{a v}\right)$ and thereby expected average ICE output power $\left(P_{e, a v}\right)$ for the planned driving route in order to determine the loading requirement of the ICE $\left(P_{e}{ }^{\prime}\right)$. Equations for the ICE output power $\left(P_{e}{ }^{\prime}\right)$ with respect to the load request $\left(P_{L}^{\prime}\right)$, available battery energy $\left(P_{\text {ess }}\right)$, and rotational moment of inertia $\left(P_{I}^{\prime}\right)$ are presented in [10].

$P_{e, a v}$ is thereby utilized by the block labeled, 'ICE Output Power Optimization" in order to calculate the required $\eta_{e, \text { low }}$ value for optimal energy balance between the ICE and ESS in satisfying current load requirements. Since the DHER controller utilizes the SOC swing charge strategy for the ESS the optimization for the ICE occurs during the ICE on phase only; hence referring to the minimization of fuel energy consumption during $t_{o n}$ in (2). For this study the total tractive energy requirement $\left(E_{\text {total }}\right)$ and estimated time of arrival $\left(t_{\text {trip }}\right)$ used to determine the average ICE output is the a priori knowledge assumed to be known at the beginning of driving for a regular trip. From the known $E_{\text {total }}$ and $t_{\text {trip }}$ ' the average tractive power requirement $\left(P_{a v}\right)$ is determined and updated as the vehicle travels towards its planned destination. This means that $E_{\text {total }}$ is reduced according to the tractive energy that is measured at the wheels of the vehicle $\left(E_{L}(t)\right.$ and therefore considered to be consumed or subtracted from the total required energy), while $t_{\text {trip }}$ ' is reduced as the vehicle approaches the 'known' time of arrival. This calculation is highlighted in equation (11). The following

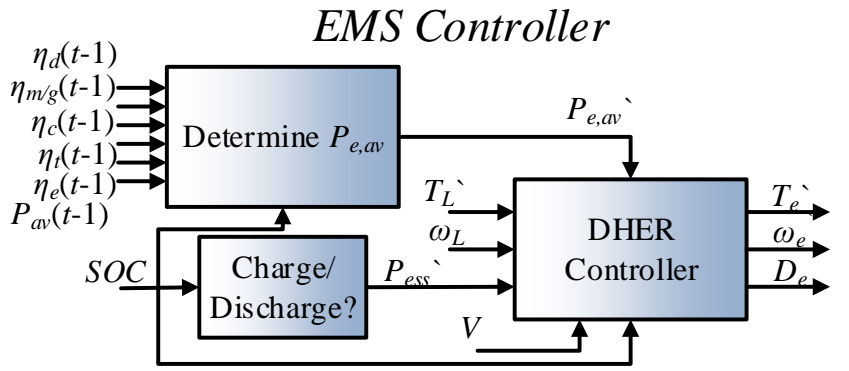

a)

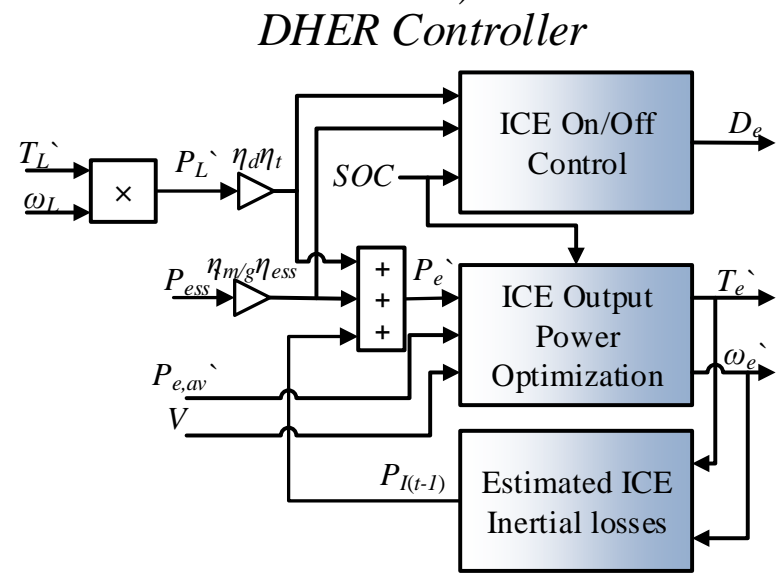

b)

Fig. 3. a) EMS controller identifying the past and present information used to determine ICE operation as a result of the DHER controller. $b$ ) Block diagram of the DHER controller outlining the ICE on/off control, and optimization of the high efficiency region $\left(\eta_{e, \text { low }}\right)$ in determining optimal ICE output power $\left(P_{e}{ }^{`}=T_{e}{ }^{`} \omega_{e}{ }^{`}\right)$.

section therefore elaborates on the fundamentals of the objective function and associated constraints identified by (2) and (3).

\section{ICE On/Off Strategy}

In HEV the single source of energy to meet drivetrain load requests is the ICE, since the ESS does not possess an external charging unit [9]. Therefore the problem for minimizing the input energy in satisfying the tractive energy requirement is dependent on the efficiency by which the fuel energy $\left(E_{f}(t)\right)$ is converted and utilized throughout the system. The above context leads to a simplified relationship between tractive energy $\left(E_{L}(t)\right)$, overall system efficiency $\left(\eta_{s y s}\right)$ and the fuel energy $\left(E_{f}(t)\right)$ consumed by the HEV for a defined period $(T)$ :

$$
E_{L}(T)=\eta_{s y s} E_{f}(T)
$$

For the considered ideal scenario, the net energy charged or discharged from the ESS is zero and therefore (4) is true if and only if the ESS energy consumed during the drive period $(T)$ is replenished at or before the end of the drive cycle:

$$
S O C_{\text {init }}=S O C_{\text {final }} \rightarrow \operatorname{SOC}(0)=S O C(T) \rightarrow E_{\text {ess }}(T)=0
$$

with zero net energy consumed by the ESS $\left(E_{\text {ess }}(t=T)=0\right)$ over the full drive period $(T)$. There are losses during the charge and discharge of the ESS which are included in the system efficiency $\left(\eta_{s y s}\right)$ and therefore energy losses are considered with respect to fuel consumption. The definition of (4) and (5) for the hybrid mode of 
operation in the HEV realizes the fundamental approach to the problem for energy management. At the fundamental level for ICE and ESS operation, maximum EMS efficiency is achieved by supplying the load with maximum ICE efficiency forcing the ESS to absorb or supply the difference [18]. This introduces the consideration for varying ICE on/off strategies to observe the benefits of intermittent ICE operation. The concern, however, is determining when to switch the ICE on and off in order to minimize fuel consumption.

Fig. 4 illustrates the expected energy balance of the load $\left(E_{L}(t)\right)$, ESS $\left(\eta_{d} \eta_{t} \eta_{m / g} \eta_{c} E_{e s s}(t)\right)$ and ICE $\left(\eta_{d} \eta_{t} \eta_{e} E_{f}(t)\right)$ at the wheels of the vehicle (Fig. 1) throughout operation. $E_{L}(t)$ is the accumulated tractive energy consumed throughout the drive period at time $t$ seconds. As mentioned $E_{L}(t)$ is subtracted from the known $E_{\text {total }}$ as the vehicle progresses throughout the planned drive scenario. Fig. 4 expands on the simplified scenario of (4) and (5) by including the losses of the powertrain and drivetrain components. Relative to the SOC swing control strategy $[5,19]$ the ESS has high and low SOC limits ( $S O C_{\text {high }}$ and $S O C_{\text {low }}$ respectively) which determines when the ICE needs to be on $\left(t_{o n}\right)$ or off $\left(t_{o f f}\right)$. This variation in energy output from the ICE $\left(\eta_{d} \eta_{t} \eta_{e} E_{f}(t)\right)$ is due to the on/off periods of the ICE and can be represented by the variable $\Delta E$ since the remaining tractive energy developed by the ICE $\left(\eta_{d} \eta_{t} \eta_{e} E_{f}(t)\right)$ is absorbed/supplied by the ESS as a result of balancing with the required tractive energy $\left(E_{L}(t)\right)$ at the wheels of the vehicle. $\Delta E$ can therefore be defined as:

$$
\Delta E=\eta_{d} \eta_{t} \eta_{m / g} \eta_{c}\left(S O C_{\text {high }}-S O C_{\text {low }}\right) E_{\text {ess,total }}
$$

where $E_{\text {ess, total }}$ is the total energy stored in the ESS [9]. If the total tractive energy requirement $\left(E_{\text {total }}{ }^{\prime}\right)$ and the time of arrival $\left(t_{\text {trip }}{ }^{\prime}\right)$ at the start of a planned route are known (or estimated from traffic conditions, driver behavior and route information [16]) then the total number and duration of ICE events are also known based on the selected SOC swing $(\triangle E)$. The selection of $S O C_{\text {high }}$ and $S O C_{\text {low }}$ depends on the desired performance outcome; prolonging battery life or minimizing fuel consumption. The Toyota Prius 2010 test bench utilizes a NiMH battery bank which is known to withstand high discharge percentages and therefore will benefit from having a large SOC swing [12]. For this reason $S O C_{\text {high }}$ and $S O C_{\text {low }}$ are selected at $80 \%$ and $40 \%$ SOC respectively, allocating a large percentage of battery energy to be available during ICE shutdown.

\section{E. Definition of the Dynamic High Efficiency Region}

Given that the ICE supplies the load at set intervals throughout a drive scenario when using the SOC swing charge strategy the objective function defined by (2) must ensure that output power developed by the ICE is maximized for minimum fuel input to the ICE. Leading on from [10], the means to achieve this is defined by selecting an optimal value of $\eta_{\text {e, low }}$ (e.g. Fig. 2) such that the tradeoff between minimizing fuel consumption and maximizing efficiency of operation is identified. For the CHER controller, selecting a high value of $\eta_{e, \text { low }}$ restricts the ICE to a higher average fuel rate consuming more fuel energy than is necessary for a given drive period. On the other hand selecting a low value of $\eta_{e, \text { low }}$ leads to inefficient ICE operation where the output power to fuel power ratio is lower than the optimal. The aim for the objective function of (2) is therefore selecting the optimal value of $\eta_{e, \text { low }}$ at the current time of operation.

To start with, consider a charging period for the EMS with $t$ residing somewhere in the $t_{o n}$ phase, as long as the SOC of the ESS

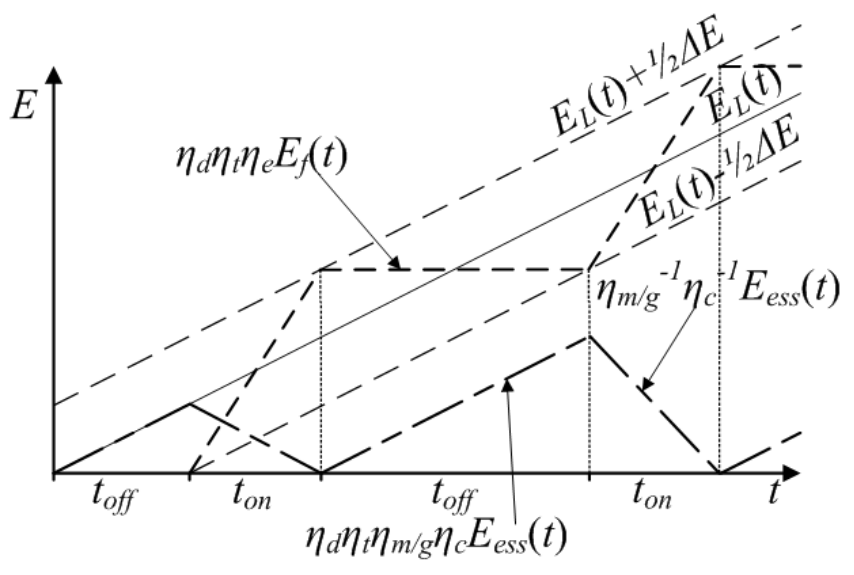

Fig. 4. Comparison of tractive energy and fuel energy consumption during driving with respect to (4) and (5).

is below the $S O C_{h i g h}$ limit the ICE will remain on. This means that for the $t_{o n}$ phase the ICE will supply an implicitly defined amount of tractive energy $\left(P_{a v}(t) t_{o n}\right)$ in addition to charging the ESS from the low limit to the high limit with the total fuel energy of:

$$
E_{f}\left(t_{o n}\right)=\frac{P_{a v}(t) t_{o n}}{\eta_{d, a v} \eta_{t, a v} \eta_{e, a v}}+\frac{\left(S O C_{h i g h}-S O C_{l o w}\right) \times E_{e s s, t o t a l}}{\eta_{e, a v} \eta_{m / g, a v} \eta_{c, a v}}
$$

where $\left(S O C_{\text {high }}-S O C_{\text {low }}\right) E_{\text {ess,total }}$ is the required charge energy at the beginning of the $t_{o n}$ phase, and each of the efficiencies identified in (7) are considered as average values for the $t_{\text {on }}$ phase. Firstly, equation (7) identifies the required optimization of the objective function in (2). Secondly, (7) identifies that in order to achieve minimum fuel consumption during $t_{\text {on }}$ the powertrain and drivetrain of the vehicle should aim to maximize the use of fuel energy during operation which is achieved by maximizing system efficiency $\left(\eta_{s y s}\right)$.

With the SOC swing control of the ESS as defined in Fig. 4 the charge and discharge of the ESS is determined according to the total driving time $\left(t_{\text {trip }}{ }^{\prime}\right)$. Using $E_{\text {total }}$ ' and the total available energy from one SOC swing (i.e $\Delta E$ ) an approximate number of charge/discharge cycles is determined $\left(f_{e}\right)$. Using the assumption of (5) $S O C_{\text {init }}$ must equal $S O C_{\text {final }}$ and therefore:

$$
f_{e}=\frac{E_{\text {total }}}{\Delta E}
$$

where $f_{e}$ is the frequency of ICE events (which is the same as the number of charge/discharge cycles). The charge/discharge period $(T)$ is then defined by an ICE on $\left(t_{o n}\right)$ and off $\left(t_{o f f}\right)$ period:

$$
t_{\text {on }}=T-t_{\text {off }}=\frac{t_{\text {trip }}}{f_{e}}-t_{\text {off }}
$$

Equation (9) ensures that regardless of the discharge time taken, the total time of the charge/discharge will be equal to $T$, calculating a minimum number of ICE events.

The minimum number of ICE events defined by (9) thereby allows for the estimation of the minimum average ICE output power requirement $P_{\eta e, \text { low }}{ }^{-}$with respect to the constraints of the objective function in (3). Due to the SOC swing charge strategy, it is expected that the ICE will remain on until the SOC of the ESS reaches the $S O C_{\text {high }}$ limit. Assuming that $S O C_{\text {init }}$ is equal to $S O C_{\text {final }}$, and the 
tractive power requirement for the load $\left(P_{L}(t)\right)$ will vary about $P_{a v}(t)$ equally over a drive cycle (Fig. 4 ), the average power required from the ICE is determined from (7) given that the total required ICE energy for the period $t_{o n}$ is known:

$$
P_{e, a v}(t)=P_{\eta e, l o w}^{-}=\frac{P_{a v}(t) t_{o n}+\Delta E}{\eta_{d, a v} \eta_{t, a v} \eta_{e, a v} \times t_{o n}}
$$

with $P_{a v}(t) t_{o n}$ being the tractive energy required during $t_{o n}$ and the SOC component $(\Delta E)$ is the energy required to recharge the ESS while the ICE remains in the on state $\left(t_{o n}\right)$. The average efficiency values of $\eta_{e}, \eta_{t}$, and $\eta_{d}$, are estimated from past driving information relative to the planned driving route or will be available as a priori information $[15,16]$ (as indicated in Fig. 3 with respect to $P_{a v}(t-1)$ and similar signals, these estimates are with respect to $t_{\text {on }}$ ). From (9) $t_{\text {on }}$ is known at the beginning of the ICE on period then following the recording of trip energy according to the constraint of (7) the ICE will switch off when $E_{f}\left(t_{o n}\right)$ exceeds $E_{L}+1 / 2 \Delta E$ which coincides with the $S O C_{\text {high }}$ limit being reached.

To ensure continuous optimal operation the total consumed tractive energy $\left(E_{L}(t)\right)$ throughout driving is used to update the total required tractive energy $\left(E_{\text {total }}{ }^{\prime}\right)$ and trip time $\left(t_{\text {trip }}{ }^{\prime}\right)$, in order to recalculate the average tractive power $\left(P_{a v}\right)$ required for the remainder of the planned trip. This ensures that any deviations from the average expected power requirement $\left(P_{a v}(t)\right.$ in $\left.(10)\right)$ are recorded and accounted for. Average tractive power is therefore equal to:

$$
P_{a v}(t)=\frac{E_{\text {total }}-E_{L}(t)}{t_{\text {trip }}-t}
$$

where $E_{L}(t)$ is the tractive energy supplied to the wheels prior to the current time $t$ throughout the drive scenario, which can be calculated as an integral of tractive power $\left(P_{L}(t)\right)$ delivered from the start of driving until time $t$. Equation (10) therefore includes the average power requirement of the drive scenario for the optimization of (2) as defined in (7) and rearranged in (10). Note that $P_{e, a v}$ of (10) (or $P_{\eta e, l o w}{ }^{-}$) is directly related to $\eta_{e, \text { low }}$ and thus the value defining the high efficiency region is calculated on-the-go.

\section{F. Implementing Dynamic High Efficiency Control}

Following the definition of the objective function and associated constraints, $\eta_{e, \text { low }}$ can be calculated with respect to the average output power requirement of the $\operatorname{ICE}\left(P_{e, a v}\right.$ in (10)). Defining a vector of $\eta_{e, \text { low }}$ values with the constraint of $\eta_{e, \max } \geq \eta_{e, \text { low }(n)}>\ldots>$ $\eta_{e, \text { low (2) }}>\eta_{e, \text { low (l) }} \geq 0$ and cross referencing these values using the optimal operating line of the ICE performance map (Fig. 2) a second vector containing ICE average power levels is established in (12).

$$
\bar{\eta}_{e, l o w}=\left[\begin{array}{c}
\eta_{e, \operatorname{low}(1)} \\
\eta_{e, l o w(2)} \\
\vdots \\
\eta_{e, l o w(n)}
\end{array}\right] \leftrightarrow \bar{P}_{e, a v}=\left[\begin{array}{c}
P_{e, a v(1)} \\
P_{e, a v(2)} \\
\vdots \\
P_{e, a v(n)}
\end{array}\right]
$$

As a result of the relationship between the efficiency contours of an ICE efficiency map and the ICE output power $\left(P_{e}\right)$ as shown in Fig. 2, each of the selected $\eta_{e, \text { low }}$ values correspond to a unique ICE output power $\left(P_{e}\right.$ or in this case $P_{e, a v}$, with $P_{\eta e, m a x} \geq P_{e, a v(n)}>\ldots>$ $\left.P_{e, a v(2)}>P_{e, a v(1)} \geq 0\right) . \bar{\eta}_{e, l o w}$ and $\bar{P}_{e, a v}$ in (12) are treated as a lookup table for the controller to convert $P_{e, a v}$ values calculated using (10)

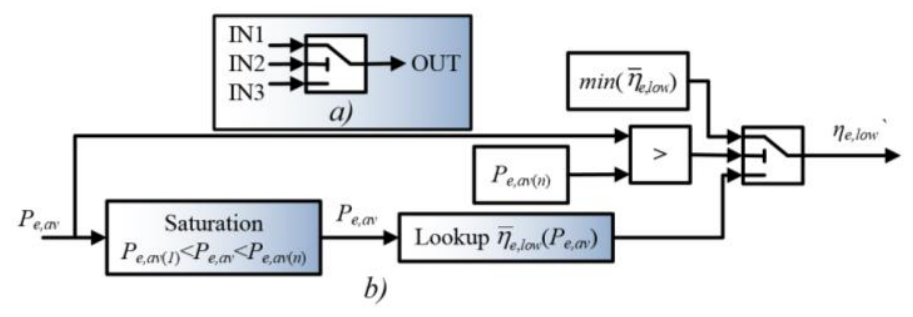

Fig. 5. a) Logical switch for signal routing. b) Selecting optimal high efficiency region for ICE operation: inserted as "Lookup $\eta_{e, \text { low }}$ " block of Fig. 6.

to $\eta_{e, l o w}$ values. $P_{e, a v}$ as stated in (10) is thereby the minimum ICE operating power that lies on the optimal operating line within the high efficiency region (i.e. $P_{\eta e, l o w}{ }^{-}$). The vectors in (12) allow the result of (10) to be fed into a look-up table to output the corresponding $\eta_{e, \text { low }}$ 'value using a method such as that shown in Fig. $5 b$ ). Firstly, the switch block of Fig. $5 b$ ) is identified in Fig. 5 $a$ ), IN1 to IN3 are the three inputs and OUT is the output. Input 2 determines whether input 1 or input 3 are passed as the output of the block. Input 2 equal to 1 will pass input 1, while input 2 equal to 0 will pass input 3 . The switch block of Fig. $5 a$ ) is also used in Fig. 6, and 7. Fig. $5 b$ ) indicates that if $P_{e, a v}$ is greater than the maximum value stored in $\bar{P}_{e, a v}$ (i.e. $\left.P_{e, a v(n)}\right)$ the ICE will remain unrestricted in order to allow maximum power to be developed by the ICE.

Once $\eta_{e, \text { low }}$ has been selected it is passed through a comparison for determining ICE requested power $\left(P_{e}{ }^{\prime}\right)$ as shown in Fig. 6. In order to minimize fuel consumption and maintain charging of the ESS at high vehicle velocity such as that employed in [10], a threshold for relocating ICE operation to the high efficiency region is determined using $V_{2}$. This means that the DHER controller will limit the charge power sent to the ESS if the SOC is greater than $S O C_{\text {init }}$ and the vehicle speed is high. The reasoning behind $V_{2}$ is to ensure that there is enough energy stored in the ESS to supply large transients at high vehicle velocity, without over charging the ESS. $V_{2}$ is selected at $18 \mathrm{~m} / \mathrm{s}$ using a similar approach to the method for $\eta_{e, \text { low }}$ selection defined in [10].

\section{G. Combined HER Control}

While the DHER controller is demonstrated to perform well over conservative drive cycles (such as highway driving) or in vehicles that have restricted driving behaviors such as public transit or company fleets, there remains a concern of how to deal with uncertainty in the event that an aggressive city drive scenario is unavoidable. Firstly, by considering the CHER and proposed DHER controllers as different modes for ICE on/off operation and switching between the two modes the advantages of both controllers are applied. This means that the high efficiency control described in Section III A to $\mathrm{F}$ in determining the varying $\eta_{e, \text { low }}$ is utilized, however, the different modes (CHER and DHER controllers) are now considered to determine when to switch the ICE on and off. For example using the CHER controller (frequent ICE on/off strategy [10]) for aggressive drive periods and the DHER controller (having SOC swing ESS charge control strategy with less frequent ICE on/off events) for conservative drive periods the results demonstrate fuel savings for all drive conditions as opposed to using either controller (CHER or DHER) as a single controller. Mode selection is determined according to Fig. 7 , with $V_{3}$ and $a_{1}$ selected to 
represent a threshold between city and highway style driving. For example $V_{3}$ selected to ensure DHER mode is selected at medium to high vehicle velocities and $a_{l}$ selected to ensure that DHER mode is selected during minimal acceleration periods as is characteristic of highway driving. Therefore, referring to Fig. $7 V_{3}$ is selected at $14 \mathrm{~m} / \mathrm{s}, a_{l}$ is selected at $0.5 \mathrm{~m} / \mathrm{s}^{2}$, and $D_{e}$ is the ICE on/off enable as identified in Fig. 3.

To summarize $V_{l}, V_{2}$ and $V_{3}$ as described in Sections III B, F and $\mathrm{G}, V_{l}$ must restrict operation of the ICE for low vehicle velocity while $V_{2}$ ensures that the ICE remains unrestricted at high vehicle velocity. In addition, $V_{3}$ must not impede upon the ability of the CHER controller to maintain frequent ICE on/off events at low vehicle velocity (i.e. $V<V_{l}$ ), or the ability of the EMS controller to enter the SOC swing charge strategy (i.e. $V>V_{2}$ ). The values for $V_{l}$, $V_{2}$ and $V_{3}$ are determined using a similar approach to the selection of $\eta_{e, \text { low }}$ in [10] with the consideration that $V_{2}>V_{3} \geq V_{l}$. As a result of the approach featured in [10] in light of the defined constraint, best performance for the Toyota Prius was determined for $V_{l}=12$ $\mathrm{m} / \mathrm{s}, V_{2}=18 \mathrm{~m} / \mathrm{s}$ and $V_{3}=14 \mathrm{~m} / \mathrm{s}$ as previously mentioned.

In order to aid the reader's understanding of the operation of the Combined HER controller, especially during DHER mode, the sequence of utilizing the aforementioned equations and block diagrams is given below. Firstly, Fig. 7 offers the selection of the ICE on/off strategy which varies based on the need to use DHER or CHER modes of operation. As mentioned $V_{3}$ and $a_{1}$ are chosen to ensure that DHER mode is selected during driving that is characteristic of highway style driving, having high vehicle velocity and minimal acceleration. Secondly, Fig. 6 identifies the final signal required to be calculated by the DHER controller as being the requested output power of the ICE $\left(P_{e}{ }^{\prime}\right) . P_{e}$ ' in Fig. 6 is, however, dependent on $P_{e, a v}$ from equation (10), therefore the controller must determine $P_{e, a v}(t)$ at any given time $t$, which begins by calculating the average load required from the drivetrain $P_{a v}$ and energy available from the ESS;

1. $P_{a v}$ in (10) is calculated from (11), with $E_{L}(t=0)=0$.

2. $\Delta E$ in (10) is determined from (6).

3. $\eta_{d}, \eta_{t}, \eta_{m / g}, \eta_{c}$ and $\eta_{e}$ are determined from past moving averages of operating efficiencies for the respective components.

4. $t_{o n}$ is determined from (9), while $f_{e}$ is calculated using (8).

5. $t_{o n}=t_{o f f}$, as a result of the assumption presented in (5).

6. With the known inputs for (10), $P_{e, a v}$ is calculated and substituted into the lookup table identified by Fig. 5 and 6. As mentioned the 'Lookup $\bar{\eta}_{e, \text { low }}\left(P_{e, a v}\right)$ ' block corresponds to equation (12), where a $P_{e, a v}$ input value is matched with the vector $\bar{P}_{e, a v}$ in order to determine the corresponding $\eta_{e, l o w}$ value.

7. $P_{e}{ }^{\prime}$ is determined from the verified simulation model of the Toyota Prius 2010 test bench [10].

8. Fig. 6 therefore determines whether to supply the requested ICE power $P_{e}$ ' or the calculated limits for operation within the high efficiency region $\left(P_{\eta e, \text { low }}{ }^{+}\right.$or $\left.P_{\eta e, \text { low }}{ }^{-}\right)$.

\section{RESULTS AND DISCUSSION}

Operation of the proposed Combined HER controller in this paper is on an HEV, such that SOC of the ESS must be maintained while minimizing fuel consumption. In order to provide a fair comparison for the simulated controllers the SOC must be corrected

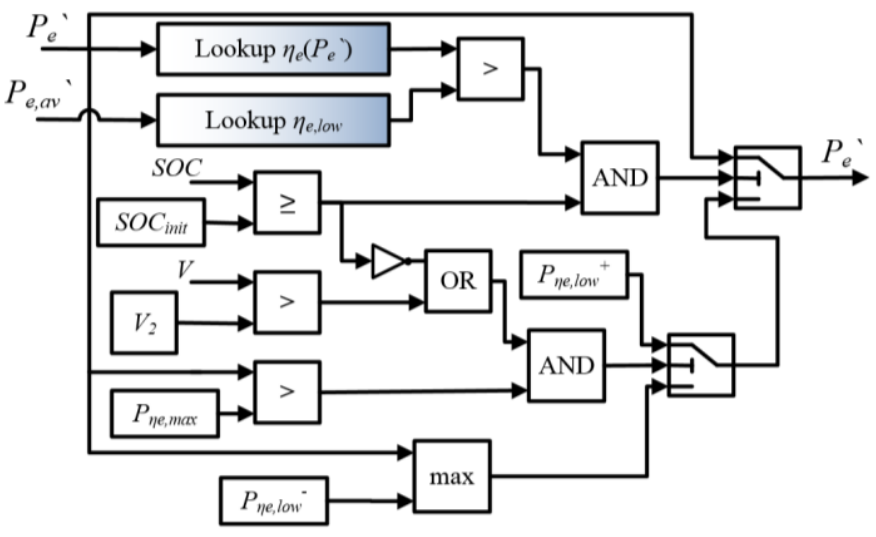

Fig. 6. Logic to determine ICE power request with respect to the determined dynamic high efficiency region. Inserted into block diagram of Fig. 3 as 'ICE Output Power Optimization'.

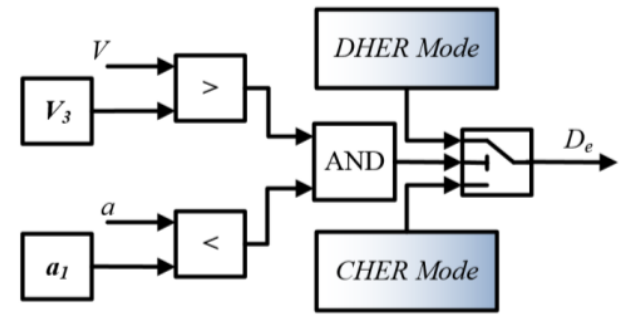

Fig. 7. Selecting mode of operation for ICE on/off to provide a single controller catering for all drive conditions.

at the end of driving. Therefore, if $S O C_{\text {final }}$ is below $S O C_{\text {init }}$, fuel energy is consumed to bring the SOC up to $S O C_{\text {init }}$ again [6]. On the other hand if $S O C_{\text {final }}$ is above $S O C_{\text {init }}$ at the end of driving no correction is required and fuel consumption is as determined in simulation. Tests are completed on a model of the Toyota Prius in simulation using the ADVISOR software as defined in [10].

The results presented in Fig. 8 of Section IV B demonstrate the ability of the Combined HER controller to restrict ICE operation to the high efficiency region for the three main drive cycles utilized in testing (UDDS, HWFET and NEDC). Then a summary of the overall performance of the controllers for the same three drive cycles is presented before examining the signals of operation for the UDDS and HWFET in more detail in Section IV C. Section IV D then evaluates the robustness of the proposed controller through the effect of erroneous a priori information on the performance of the proposed controller. Firstly, however, there are concerns for the operation of the Combined HER controller during ICE shutdown as seen on the selected test bench.

\section{A. SOC Swing Control Limitations}

The maximum discharge power of the ESS in the Toyota Prius 2010 test bench is not large enough to support operations during ICE shutdown [20]. This limitation is highlighted by the definition of $P_{\text {ess, } \max }$ in (13):

$$
P_{e s s, \max }=\frac{\left(V_{o c}-V_{e s s, \min }\right) V_{e s s, \text { min }}}{R_{\mathrm{int}}}
$$

where $P_{\text {ess,max }}$ is the maximum discharge power of the ESS, $V_{o c}$ the open circuit voltage, $V_{\text {ess, min }}$ the minimum ESS voltage and $R_{\text {int }}$ the internal resistance during discharge. Equation (13) highlights that if the ESS $V_{o c}$ is low then $P_{e s s, \max }$ will also be restricted, which 
is the case for the Toyota Prius 2010. Increasing $V_{o c}$ of the ESS increases the maximum discharge power allowed from the ESS according to (13), however, it also increases the energy available in the ESS and therefore may affect performance. For the considered test bench simulations are completed with a $V_{o c}$ scaled by 1.4 , effectively increasing the stored energy by $40 \%$. The effects on the performance of the CHER and original controllers realize increased fuel savings due to increased availability of energy from the ESS. This is in comparison to the results realized in [10]. Comparison of the three controllers using the increased $V_{o c}$ for the ESS demonstrates the advantages and disadvantages of using the proposed Combined HER controller.

\section{B. Restricting ICE Operation On-The-Go}

During ICE operation the Combined HER controller utilizes the defined DHER control to ensure optimal ICE output power for the required load demand. $\eta_{e, \text { low }}$ is the control signal determining ICE minimum operating efficiency (defined in Section III E and F). Fig. 8 displays the calculated value of $\eta_{e, \text { low }}$ using (10) to determine $P_{e, a v}$ and (12) to lookup the corresponding $\eta_{e, \text { low }}$ value. The $\eta_{e, \text { low }}$ value of Fig. 8 is resulting from the DHER control approach and is substituted into the "Lookup $\eta_{e, \text { low" }}$ " control process of Fig. 6. Fig. 8 indicates that no restrictions are placed on the ICE during the HWFET since the requested power exceeds the $P_{e, a v(n)}$ value identified in (12), however, for both the UDDS and NEDC drive profiles that have $P_{e, a v}$ values within the defined range $\left(P_{e, a v(I)}\right.$ to $\left.P_{e, a v(n)}\right)$ there exists a corresponding $\eta_{e, \text { low }}$ value. It is also noted that any benefit observed for the HWFET drive profile is a result of identifying the need to operate the ESS charge/discharge according to the SOC swing ESS control strategy as opposed to restricting ICE operation to the high efficiency region. Fig. 8 thereby demonstrates the potential for utilizing the DHER control of the ICE in the Combined HER controller to autonomously restrict ICE operation. In the case of the CHER controller ICE operation is restricted to the two constant $\eta_{e, \text { low }}$ values, $\eta_{e, \text { low } 1}=20 \%$ and $\eta_{e, \text { low } 2}$ $=28 \%$ only.

\section{Performance Comparison}

Table II summarizes the performance of the CHER, DHER and Combined HER controllers on the Toyota Prius 2010 test bench with the original controller used as the bench mark for fuel consumption improvement calculations. SOC correction of the final fuel consumption for each case has taken place to ensure that the results will be maintained for future drive conditions. The comparison presented in Table II identifies the advantages of using either the CHER or DHER controller, and then the resulting improvement observed in the Combined HER controller. Firstly, with respect to the drivability, the DHER controller demonstrates a very low number of ICE events over each of the drive cycles since the controller has calculated the optimal time periods to recharge the ESS [8]. Impacts of the CHER controller on drivability is discussed in [10], however, for the considered comparison a high number of ICE events is undesirable. Secondly, the use of the DHER controller during highly transient drive cycles such as the UDDS reveal increased fuel consumption, as identified in Table II at 3.63 $\mathrm{L} / 100 \mathrm{~km}$ compared to $3.24 \mathrm{~L} / 100 \mathrm{~km}$ for the original controller. On the other hand the operation of the Toyota Prius using the SOC swing ESS charge strategy (as implemented in the DHER controller) has benefits for less aggressive (or conservative) drive profiles such as the HWFET and NEDC. While there are some large

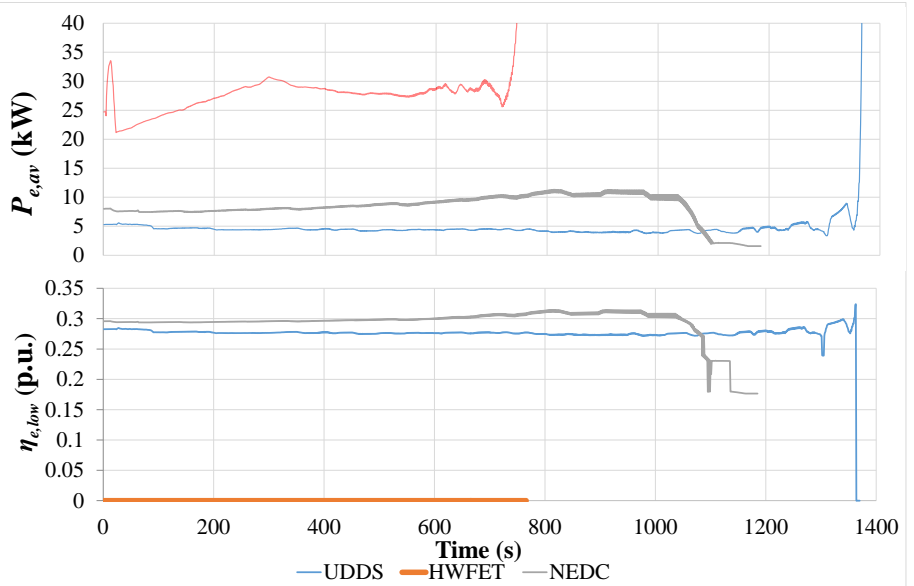

Fig. 8. Restricting ICE operation to the high efficiency region; calculating $P_{e, a v}$ to determine $\eta_{e, \text { low }}$ on-the-go.

TABLE II

PERFORMANCE COMPARISON WITH $1.4 V_{O C}$

\begin{tabular}{|c|c|c|c|c|c|}
\hline \multirow{2}{*}{$\begin{array}{l}\text { Dri. } \\
\text { Cyc. }\end{array}$} & \multirow{2}{*}{ Component Parameter } & \multicolumn{4}{|c|}{ Toyota Prius 2010} \\
\hline & & Orig. & CHER & DHER & Combined \\
\hline \multirow{10}{*}{$\tilde{\hat{⿵}}$} & ESS Initial SOC (\%) & 60 & 60 & 60 & 60 \\
\hline & ESS Final SOC (\%) & 60 & 60 & 65.69 & 60 \\
\hline & ICE Events & 56 & 59 & 43 & 54 \\
\hline & Avg ICE Eff. (\%) & 28.57 & 31.71 & 31.84 & 31.39 \\
\hline & Avg Mot Eff. (\%) & 81.14 & 83.43 & 85.22 & 85.1 \\
\hline & Avg Gen Eff. (\%) & 84.74 & 85.59 & 87.62 & 84.82 \\
\hline & Avg ESS RT Eff. (\%) & 85.87 & 87.76 & 77.71 & 84.59 \\
\hline & Overall Eff. (\%) & 10.12 & 10.90 & 8.45 & 10.84 \\
\hline & Fuel Cons. (L/100km) & 3.24 & 2.96 & 3.63 & 3.08 \\
\hline & FC Imp. (\%) & N/A & 8.64 & -12.04 & 4.94 \\
\hline \multirow{10}{*}{ 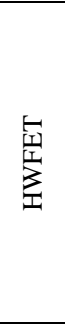 } & ESS Initial SOC (\%) & 60 & 60 & 60 & 60 \\
\hline & ESS Final SOC $(\%)$ & 60 & 60.79 & 60 & 60 \\
\hline & ICE Events & 5 & 5 & 1 & 2 \\
\hline & Avg ICE Eff. (\%) & 27.29 & 29.91 & 31.92 & 31.91 \\
\hline & Avg Mot Eff. (\%) & 77.93 & 86.26 & 90.89 & 91.14 \\
\hline & Avg Gen Eff. (\%) & 89.52 & 90.05 & 91.26 & 91.1 \\
\hline & Avg ESS RT Eff. (\%) & 90.49 & 90.79 & 85.80 & 86.66 \\
\hline & Overall Eff. (\%) & 19.00 & 20.39 & 20.86 & 21.12 \\
\hline & Fuel Cons. (L/100km) & 3.35 & 3.05 & 2.97 & 2.96 \\
\hline & FC Imp. $(\%)$ & N/A & 8.96 & 11.34 & 11.64 \\
\hline \multirow{10}{*}{ 岂 } & ESS Initial SOC (\%) & 60 & 60 & 60 & 60 \\
\hline & ESS Final SOC (\%) & 60 & 61.96 & 60 & 60 \\
\hline & ICE Events & 11 & 16 & 1 & 12 \\
\hline & Avg ICE Eff. (\%) & 26.49 & 30.13 & 33.59 & 32.07 \\
\hline & Avg Mot Eff. (\%) & 75.93 & 76.2 & 84.77 & 86.66 \\
\hline & Avg Gen Eff. (\%) & 85.66 & 86.72 & 90.04 & 89.15 \\
\hline & Avg ESS RT Eff. (\%) & 90.34 & 90.08 & 84.78 & 83.34 \\
\hline & Overall Eff. (\%) & 13.45 & 14.42 & 14.88 & 16.02 \\
\hline & Fuel Cons. (L/100km) & 3.55 & 3.22 & 3.12 & 3.13 \\
\hline & FC Imp. $(\%)$ & N/A & 9.30 & 12.11 & 11.83 \\
\hline
\end{tabular}

Avg ESS Round Trip Eff. (\%) has been shortened to Avg ESS RT Eff. (\%).

transients required for acceleration of the vehicle during ICE shutdown on the HWFET and NEDC drive cycles, such requests are less frequent than those required on the UDDS. The DHER controller performs between $2 \%$ and $3 \%$ better than the CHER controller on the HWFET and NEDC respectively. This demonstrates that the preferred use for the DHER controller is during highway style driving where large transients are less frequent, or city driving where vehicle speeds are relatively constant and acceleration requirements are low.

The resulting performance of the Combined HER controller as listed in Table II highlights that fuel consumption reductions are achieved for all drive cycles, having utilized the advantages of both of the CHER and DHER controllers. From Table II the Combined 
TABLE III

ERROR IN FINAL FUEL CONSUMPTION DUE TO INACCURACY OF A PRIORI INFORMATION

\begin{tabular}{lcc}
\hline \hline $\begin{array}{c}\text { Component } \\
\text { Parameter }\end{array}$ & MAPE $(\%)$ & $\sigma^{2}(\mathrm{~L} / 100 \mathrm{~km})$ \\
\hline$E_{\text {total }} \pm 10 \%$ & 0.63 & 0.019 \\
$E_{\text {total }} \pm 20 \%$ & 1.06 & 0.034 \\
$t_{\text {trip }} \pm 10 \%$ & 0.97 & 0.030 \\
$t_{\text {trip }} \pm 20 \%$ & 1.27 & 0.040 \\
\hline \hline
\end{tabular}

HER controller is achieving $11.64 \%$ and $11.83 \%$ improvement to fuel consumption over the HWFET and NEDC, drive cycles which is similar to the DHER controller. The results do however, acknowledge that there is a compromise between the CHER control and Combined HER control for city drive scenarios since the CHER controller is having greater fuel savings at $8.64 \%$ improvement compared to $4.94 \%$ respectively for the UDDS. Additionally, the mode selection for each drive scenario is partially identified by the number of ICE events shown in Table II, such that a high number of ICE events is a result of the CHER ICE on/off control being selected for the majority of driving while a low number of ICE events is representative of the SOC swing ESS charge strategy control being selected.

Referring to Fig. 9 the resulting output signals of the Toyota Prius using both the CHER and Combined HER controllers are shown for continuous operation over the UDDS and HWFET. Demonstrating operation over the two drive cycles distinguishes the performance of the Combined HER controller for city and highway driving. $\Delta t_{a}$ identifies a discharge period during DHER mode of the Combined HER controller (Fig. 9b), which avoids fossil fuel consumption that is otherwise utilized during the CHER controller, however, before the energy can be replenished in DHER mode the controller switches to CHER mode in order to meet the highly transient load demand. In this period the difference between the CHER and Combined HER controllers is that DHER mode forces the ICE to switch off and ESS energy is utilized to satisfy the load. The restriction for reducing fossil fuel consumption in the UDDS is a result of having to switch to the CHER mode in order to meet the load demand. Therefore the full benefit of the DHER controller is restricted. $\Delta t_{b}$ on the other hand observes a period of CHER mode for the Combined HER controller as a result of the highly transient load demand. Some ESS energy is replenished due to the CHER charging strategy during this mode of operation, however, both the CHER and Combined HER controllers are operating very similarly during this period.

$\Delta t_{c}$ and $\Delta t_{d}$ highlight the full benefit of utilizing the DHER mode, where ESS energy is utilized to meet the demand during $\Delta t_{c}$ followed by the ICE having an increased load in order to replenish discharged ESS energy during $\Delta t_{d}$. For $\Delta t_{c}$ the benefit of the DHER mode is realized by avoiding ICE fuel consumption and utilizing energy from the highly efficient ESS and M/G combination. Then for $\Delta t_{d}$ ESS energy is recharged during high efficiency operation of the ICE with only a minor increase in the fuel rate. The ability of the DHER mode to discharge and then efficiently recharge the ESS while the ICE is meeting load demand ensures an efficient use of fossil energy. The increase in average ICE efficiency as identified in Table II with a comparison between the CHER and Combined HER controllers, summarizes the performance for the DHER mode and in particular $\Delta t_{d}$ (i.e. CHER at $29.91 \%$ and Combined HER at $31.91 \%$ for the HWFET).

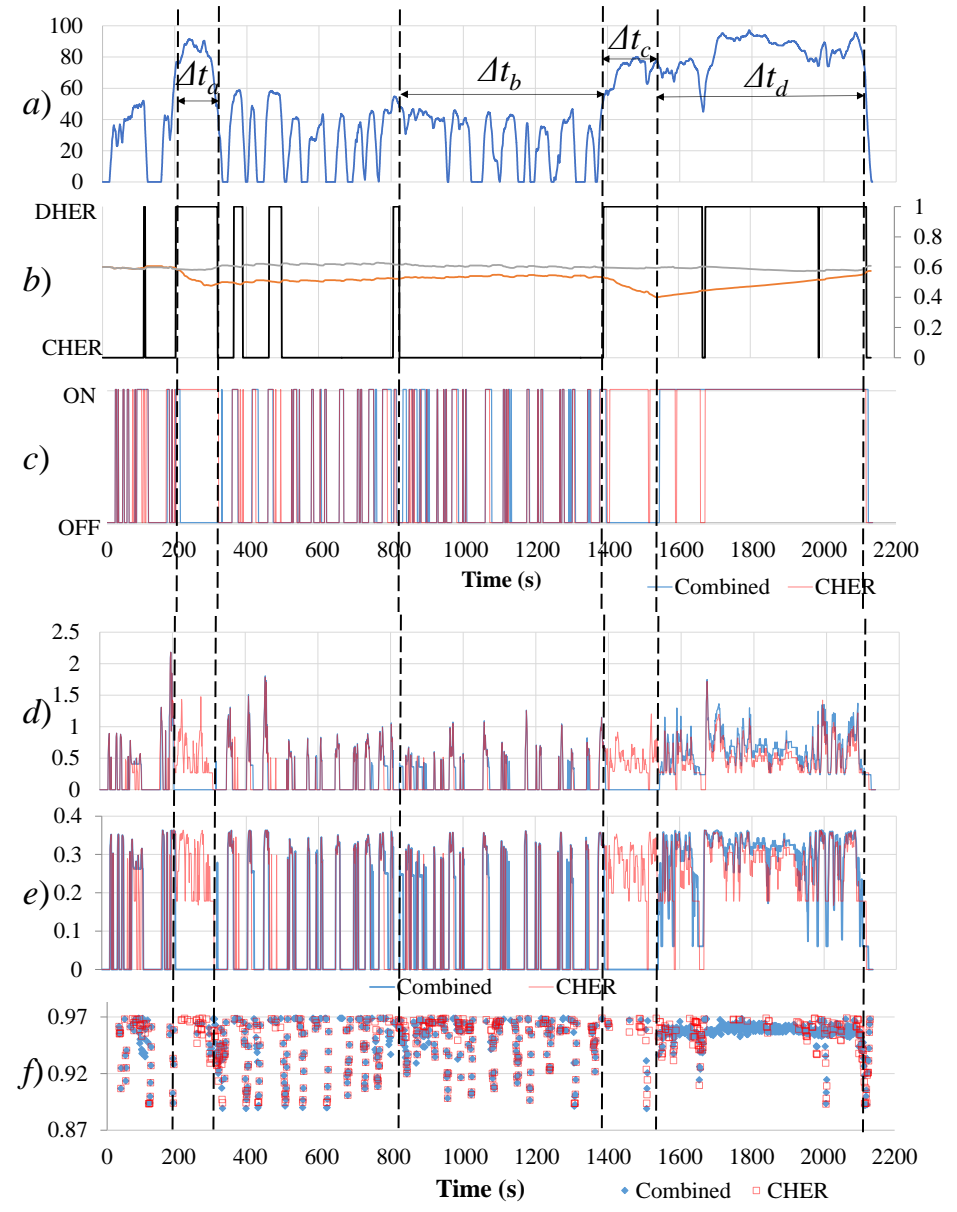

Fig. 9. Comparison of signals for operation of the Combined HER and CHER controllers over the UDDS and HWFET drive cycles. $a)$ Drive cycle $(\mathrm{km} / \mathrm{h}), b)$ Combined HER controller mode select (primary axis), ESS SOC (p.u.) (secondary axis), $c$ ) ICE on/off signal, $d$ ) ICE fuel rate (g/s), e) ICE efficiency (p.u.), $f$ ) ESS charging efficiency (p.u.).

The results of Table II in combination with Fig. 9 thereby demonstrate the benefits for autonomous high efficiency control in combination with SOC swing ESS charge strategy control (Combined HER control) during conservative drive scenarios and the potential for mode selection based on the requirements of driving.

\section{Error Tolerance for Previewed Information}

With the consideration for a priori knowledge as determined from ITS such as that featured in [15], the proposed controller assumes knowledge of the exact total tractive energy $\left(E_{\text {total }}{ }^{\prime}\right)$ and time of arrival $\left(t_{\text {trip }}{ }^{\prime}\right)$. In order to demonstrate the impact, errors in calculating $E_{\text {total }}$ ' and $t_{\text {trip }}$ ' may have on the proposed controller's performance, a percentage variation is introduced in simulation. For each of the tested drive cycles the results are combined in Table III, which identifies the mean absolute percentage error (MAPE, \%) [21] and variance $\left(\sigma^{2}\right)$ in fuel consumption resulting from an error of up to $20 \%$ in $E_{\text {total }}$ and $t_{\text {trip }}$. The MAPE demonstrates a percentage difference between the fuel consumption achieved using the exact $E_{\text {total }}$ ' or $t_{\text {trip }}{ }^{\prime}$ values and the identified percentage errors $( \pm 10 \%$ and $\pm 20 \%$ ), while the variance identifies an approximate change in the quantity of fuel in L/100km. Table III indicates that with a $\pm 20 \%$ error in $t_{\text {trip }}$ ' the fuel consumption may increase by $1.27 \%$, for example on the HWFET using the proposed Combined 
HER controller (Table II) this is an increase from $2.96 \mathrm{~L} / 100 \mathrm{~km}$ to $3.00 \mathrm{~L} / 100 \mathrm{~km}$, which is a minor variation. The results of Table III highlight the robust nature of the proposed controller such that once a reasonable estimate of the driving requirements for a planned route is achieved the expected fuel savings are consistent.

\section{CONCLUSION}

This paper proposes an improvement to the recently developed constant high efficiency region (CHER) controller by introducing a dynamically varying high efficiency region for operation of the ICE in combination with a SOC swing ESS charge control strategy. The proposed controller is referred to as the Combined HER controller and it relies on a priori knowledge of the total energy and trip time for a given drive scenario. Such information is available from ITS having the capability to preview information related to traffic conditions, driver behavior and planned driving routes. This allows the controller to calculate the expected charge and discharge cycles of the ESS in advance based on the available stored energy of the battery. The outcome is a reduced number of ICE events with the potential for fuel savings in conservative city drive scenarios or highway drive scenarios. In addition, the proposed controller improves on the CHER controller ICE operation by introducing autonomous calculation of the high efficiency region $\left(\eta_{e, \text { low }}\right)$.

\section{REFERENCES}

[1] U.S. Energy Information Administration (EIA), "International Energy Outlook 2013," Office of Energy Analysis, U.S. Department of Energy, Washington, DC, Jul. 2013.

[2] U.S. Department of Energy. (2013, 15 July 2013). Download Fuel Economy Data. Available: http://www.fueleconomy.gov/feg/download.shtml

[3] S. G. Wirasingha, R. Gremban, \& A. Emadi, "Source-to-Wheel (STW) Analysis of Plug-in Hybrid Electric Vehicles," Smart Grid, IEEE Transactions on, vol. PP, pp. 1-1, 2011.

[4] V. Sezer, M. Gokasan, and S. Bogosyan, "A Novel ECMS and Combined Cost Map Approach for High-Efficiency Series Hybrid Electric Vehicles," Vehicular Technology, IEEE Transactions on, vol. 60, pp. 3557-3570, 2011.

[5] K. Hayasaki, T. Abe, K. Tanishima, \& K. Chujo "Development of a Parallel Hybrid System for RWD Vehicles," in Advanced Hybrid Vehicle Powertrains, World Congress, SAE, Detroit, MI, Paper 2011-01-0884, 2011.

[6] S. J. Moura, H. K. Fathy, D. S. Callaway, \& J. L. Stein, "A stochastic optimal control approach for power management in plug-In hybrid electric vehicles," Control Systems Technology, IEEE Transactions on, vol. 19, pp. 545-555, 2011.

[7] H. Borhan, A. Vahidi, A. M. Phillips, M. L. Kuang, I. V. Kolmanovsky et al., "MPC-Based Energy Management of a Power-Split Hybrid Electric Vehicle," Control Systems Technology, IEEE Transactions on, vol. PP, pp. 1-11, 2011.

[8] D. F. Opila, X. Wang, R. McGee, R. B. Gillespie, J. A. Cook, and J. W. Grizzle, "An Energy Management Controller to Optimally Trade Off Fuel Economy and Drivability for Hybrid Vehicles," Control Systems Technology, IEEE Transactions on, vol. 20, pp. 1490-1505, 2012.

[9] M. Ehsani, Y. Gao, \& A. Emadi, Modern electric, hybrid electric, and fuel cell vehicles : fundamentals, theory, and design, 2nd ed. Boca Raton: CRC Press, 2010.

[10] S. Overington and S. Rajakaruna, "High Efficiency Control of Internal Combustion Engines in Blended Charge Depletion/Charge Sustenance Strategies for Plug-in Hybrid Electric Vehicles," Vehicular Technology, IEEE Transactions on, vol. PP, pp. 1-1, 2014.

[11] S. J. Moura, J. L. Stein, and H. K. Fathy, "Battery-Health Conscious Power Management in Plug-In Hybrid Electric Vehicles via Electrochemical Modeling and Stochastic Control," Control Systems Technology, IEEE Transactions on, vol. 21, pp. 679-694, 2013.

[12] A. F. Burke, "Batteries and Ultracapacitors for Electric, Hybrid, and Fuel Cell Vehicles," Proceedings of the IEEE, vol. 95, pp. 806-820, 2007.

[13] J. T. B. A. Kessels, M. W. T. Koot, P. P. J. van den Bosch, \& D. B. Kok, "Online Energy Management for Hybrid Electric Vehicles," Vehicular Technology, IEEE Transactions on, vol. 57, pp. 3428-3440, 2008.
[14] C. C. Chan, A. Bouscayrol, \& K. Chen, "Electric, Hybrid, and Fuel-Cell Vehicles: Architectures and Modeling," IEEE Trans. on Vehicular Technology, vol. 59, pp. 589-598, Feb. 2010.

[15] G. Qiuming, L. Yaoyu, \& P. Zhong-Ren, "Trip-Based Optimal Power Management of Plug-in Hybrid Electric Vehicles," Vehicular Technology, IEEE Transactions on, vol. 57, pp. 3393-3401, 2008.

[16] Z. Chen, \& A. Vahidi, "Route Preview in Energy Management of Plug-in Hybrid Vehicles," Control Systems Technology, IEEE Transactions on, vol. 20, pp. 546-553, 2012.

[17] P. Tulpule, V. Marano, G. Rizzoni, R. McGee, \& H. Yu, "A Statistical Approach to Assess the Impact of Road Events on PHEV Performance using Real World Data," in Advanced Hybrid Vehicle Powertrains, World Congress, SAE, Detroit, MI, Paper 2011-01-0875, 2011.

[18] S. G. Wirasingha, \& A. Emadi, "Classification and Review of Control Strategies for Plug-In Hybrid Electric Vehicles," Vehicular Technology, IEEE Transactions on, vol. 60, pp. 111-122, 2011.

[19] S. S. Raghavan and A. Khaligh, "Electrification Potential Factor: EnergyBased Value Proposition Analysis of Plug-In Hybrid Electric Vehicles," Vehicular Technology, IEEE Transactions on, vol. 61, pp. 1052-1059, 2012.

[20] G. L. Plett, "High-performance battery-pack power estimation using a dynamic cell model," Vehicular Technology, IEEE Transactions on, vol. 53, pp. 15861593, 2004.

21] N. Daiheng, \& D. Henclewood, "Simple Engine Models for VII-Enabled InVehicle Applications," Vehicular Technology, IEEE Transactions on, vol. 57, pp. 2695-2702, 2008.

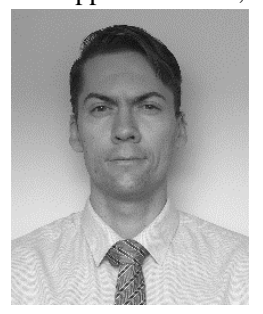

Shane Overington (S'08-M'10) received the degrees BEng (Hons) and PhD from Curtin University, Perth, Western Australia, Australia, in 2010 and 2015, respectively, both in the area of electrical engineering. He is currently a University Associate at Curtin University while working full time for GET Trakka Pty Ltd as an Engineering Analyst. His role at GET Trakka Pty Ltd focusses on the improvement of their wireless sensor tag solution for breakage detection of ground engaging tools (GET) on mine site diggers. He is a recipient of the Australian Postgraduate Award (APA) scholarship and Curtin University Postgraduate Scholarship (CUPS) having achieved first class honors during his undergraduate studies. His research interests include control systems, energy management, renewable energy, and smart vehicles.

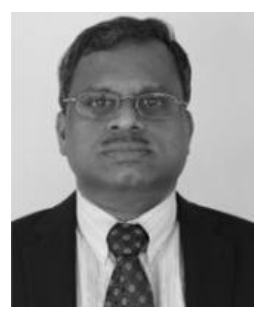

Sumedha Rajakaruna (M'93-SM'06) received the degrees B.Sc.Eng., M.Sc. and Ph.D., from University of Moratuwa, Sri Lanka in 1986, University of Calgary, Canada in 1989 and University of Toronto, Canada in 1993, respectively, all in the area of electrical engineering. From 1993 to 2000, he was a Senior Lecturer with the Department of Electrical Engineering, University of Moratuwa, Sri Lanka. From 2000 to 2007, he was at the School of Electrical and Electronic Engineering, Nanyang Technological University, Singapore as an Assistant Professor. Since 2007, he is working as a Senior Lecturer at the department of Electrical and Computer Engineering, Curtin University, Australia. He is also the Founding Director of Green Electric Energy Park at Curtin University. His present research interests include renewable energy, microgrids, smart vehicles and power electronics. 